\title{
Causal Theory of Relativistic Dissipative Hydrodynamics
}

\author{
G. S. Denicol, T. Kodama, T. Koide, and Ph. Mota \\ Instituto de Física, Universidade Federal do Rio de Janeiro, C. P. 68528, 21945-970, Rio de Janeiro, Brazil
}

Received on 10 November, 2006

\begin{abstract}
We present a new formalism for the theory of relativistic dissipative hydrodynamics, where covariance and causality are satisfied by introducing the memory effect in irreversible currents. Our theory has a much simpler structure and thus has several advantages for practical purposes compared to the Israel-Stewart theory (IS). We apply our formalism to the Bjorken model and the results are shown to be analogous to the IS.
\end{abstract}

Keywords: Causality; Dissipation; Hydrodynamics

\section{INTRODUCTION}

The ideal hydrodynamical description for the dynamics of hot and dense matter achieved in RHIC experiments works amazingly well, particularly for the behavior of collective flow parameters. Together with other signals, the success of the approach is considered as the indication of the emergence of a new state of strongly interacting matter, the plasma of quarks and gluons (QGP). The comparison between RHIC and SPS results shows that this new state of matter is formed at the very early stage of the relativistic heavy ion collisions for RHIC energies.

However, we know that there still exist several open problems in the interpretation of data in terms of the hydrodynamical model [1]. These questions require careful examination to extract quantitative and precise information on the properties of QGP. In particular, we should study the effect of dissipative processes on collective flow variables. Several works have been done in this direction [2]. However, strictly speaking, a quantitative and consistent analysis of the viscosity within the framework of relativistic hydrodynamics has not yet been done completely. This is because the introduction of dissipative phenomena in relativistic hydrodynamics casts difficult problems, both conceptual and technical. Initially Eckart, and later, Landau-Lifshitz introduced the dissipative effects in relativistic hydrodynamics in a covariant manner [3, 4]. It is, however, known that their formalism leads to the problem of non-causality, that is, a pulse signal propagates with infinite speed. Thus, relativistic covariance is not a sufficient condition for a consistent relativistic dissipative dynamics $[5,6]$.

To cure this problem, relativistic hydrodynamics in the framework of extended thermodynamics was developed by Müller [7] and later by Israel and Stewart [8-10]. From the kinetic point of view [11], this formalism corresponds to the extension of equilibrium thermodynamics to include the second order moments of kinetic variables. This is the reason why this theory is usually refereed to as the second order theory. However, it is known that the Israel-Stewart theory (IS) is not the unique approach to relativistic dissipative hydrodynamics. To the authors' knowledge, there is at least one other causal theory called the divergence type [12-16]. In this work, we present a very simple alternative theory which satisfies the minimal conditions mentioned above. We show that the causality problem of the Landau-Lifshitz formalism (LL) can be solved by introducing a memory effect. This memory effect is characterized by the relaxation time $\tau_{R}$, so that our theory introduces only one additional parameter to the usual bulk viscosity, shear viscosity and thermal conduction coefficients of the Navier-Stokes equation. Our theory recovers the relativistic Navier-Stokes equation in the limit of vanishing values of this relaxation time.

As described later more in detail, our approach has a fundamental advantage from the practical point of view in addition to its physical simplicity. The dissipative terms are explicitly given by the integral of the independent variables of the usual ideal hydrodynamics. Thus, the implementation of our method to the existing ideal hydro-codes is straightforward, particularly, to those based on the local Lagrangian coordinate system such as SPheRIO [17, 18].

The present paper is organized as follows. In the next section, we briefly review the problem of non-causal propagation in the diffusion equation and the method to cure this problem in terms of the memory effect, which leads to the so-called telegraphist's equation. In the Sec. III, we analyze the structure of the LL of relativistic dissipative hydrodynamics and introduce the memory effect to solve the non-causal problem due to its parabolic nature. We thus obtain the dissipative hydrodynamical equations with the minimum number of parameters which satisfies causality. In Sec. IV, we discuss the problem of entropy production in our formalism. In Sec. V, we discuss the propagation speed of our equation. In Sec.VI, we apply our equation to the Bjorken solution, and compare with the previous analysis [19-23]. In Sec. VII, we summarize our results and discuss possible immediate applications.

\section{DIFFUSION EQUATION AND ACAUSALITY}

The fundamental problem of first-order theories, like the Navier-Stokes theory, is attributed to the fact that the diffusion equation is parabolic. The diffusion process is a typical relaxation process of conserved quantities. Thus, it should satisfy the equation of continuity,

$$
\frac{\partial n}{\partial t}+\nabla \cdot \vec{j}=0
$$

where $n$ is a density of a conserved quantity. The irreversible current $\vec{j}$ is, phenomenologically assumed to be proportional to a thermodynamic force $F$, which is given by the gradient of 
$n$,

$$
\vec{j}=-\zeta \vec{F}=-\zeta \nabla n,
$$

where the Onsager coefficient $\zeta$. Substituting Eq.(2) into Eq.(1), we get the diffusion equation,

$$
\frac{\partial}{\partial t} n=\zeta \nabla^{2} n .
$$

Fick's law tells us that the above diffusion process is induced by an inhomogeneous distribution. In Eq.(2), the space inhomogeneity immediately gives rise to the irreversible current. However, this is a very idealized case. In general, the generation of irreversible currents has a time delay. Thus, we may think of memory effects by introducing the following memory function [26-28],

$$
\begin{array}{rlr}
G\left(t, t^{\prime}\right) & =\frac{1}{\tau_{R}} e^{-\left(t-t^{\prime}\right) / \tau_{R}}, t \geq t^{\prime} \\
& =0, & t<t^{\prime}
\end{array}
$$

where $\tau_{R}$ characterizes the memory time and called the relaxation time. Then, we rewrite Eq.(2) as

$$
\vec{j}=-\int_{-\infty}^{t} G\left(t, t^{\prime}\right) \zeta \vec{F}\left(t^{\prime}\right) d t^{\prime} .
$$

In the limit of $\tau_{R} \rightarrow 0$, we have $G\left(t, t^{\prime}\right) \rightarrow \delta\left(t-t^{\prime}\right)$ so that the original equation (2) is recovered [29]. Substituting into the equation of continuity (1), we arrive at

$$
\tau_{R} \frac{\partial^{2} n}{\partial t^{2}}=-\frac{\partial n}{\partial t}+\zeta \nabla^{2} n .
$$

This equation is hyperbolic. This telegraph equation is sometimes called the causal diffusion equation.

The maximum velocity of the signal propagation of the causal diffusion equation is [25],

$$
v_{\max }=\sqrt{\frac{\zeta}{\tau_{R}}} .
$$

For a suitable choice of the parameters $\tau_{R}$ and $\zeta$, we can recover causal propagation of the diffusion process. On the other hand, the diffusion equation corresponds to $\tau_{R}=0$ and hence $v_{\max } \rightarrow \infty$. This is the reason why the diffusion equation breaks causality.

\section{RELATIVISTIC DISSIPATIVE HYDRODYNAMICS}

Eckart and Landau-Lifshitz derived relativistic dissipative hydrodynamics following non-equilibrium thermodynamics as discussed in the preceding section $[3,4]$. Their theories are just the covariant versions of the Navier-Stokes equation and the corresponding equations still continue to be parabolic. Therefore, they do not satisfy causality and some modification should be required. In the IS and the divergence type theory, the definition of the entropy four-flux is generalized and, to satisfy the second law of thermodynamics, modified thermodynamic forces are obtained. In this section, we propose another approach, where the problem of non-causality is solved by introducing the memory effect as was done in Eq. (5) [30].

For this purpose, let us first analyze briefly the structure of the LL. The hydrodynamical equation of motion is written as the conservation of the energy-momentum tensor,

$$
\partial_{\mu} T^{\mu v}=0,
$$

together with the conservation of a quantity, for example, the baryon number,

$$
\partial_{\mu} N^{\mu}=0
$$

In the LL, it is assumed that thermodynamic relations are valid in the local rest frame of the energy-momentum tensor. The energy-momentum tensor is expressed as

$$
T^{\mu v}=\varepsilon u^{\mu} u^{v}-P^{\mu v}(p+\Pi)+\pi^{\mu v},
$$

where, $\varepsilon, p, u^{\mu}, \Pi$ and $\pi^{\mu v}$ are respectively the energy density, pressure, four velocity of the fluid, bulk viscosity and shear viscosity. In the LL, the velocity field is defined in such a way that the energy current vanishes in the local rest frame, $u^{\mu} \rightarrow(1,0,0,0)$. In this local rest frame, it is assumed that the equation of state and thermodynamical relations are valid as if it were in equilibrium. As usual, we write

$$
u^{\mu}=\left(\begin{array}{c}
\gamma \\
\gamma \vec{v}
\end{array}\right),
$$

where $\gamma$ is the Lorentz factor and

$$
u^{\mu} u_{\mu}=1
$$

The tensor $P^{\mu v}$ is the projection operator to the space orthogonal to $u^{\mu}$ and given by

$$
P^{\mu v}=g^{\mu v}-u^{\mu} u^{v} .
$$

In the LL, the current for the conserved quantity (e.g., baryon number) takes the form

$$
N^{\mu}=n u^{\mu}+v^{\mu},
$$

where $v^{\mu}$ is the heat conduction part of the current. For the irreversible currents, we require the constraints [4],

$$
u_{\mu} \pi^{\mu \nu}=0
$$

and

$$
u_{\mu} v^{\mu}=0 .
$$

These constraints permit us to interpret $\varepsilon$ and $n$ respectively as the energy and baryon number densities in the local rest frame. In fact, from Eq.(13), in the rest frame, we have

$$
N^{\mu} \rightarrow\left(\begin{array}{c}
n \\
\vec{v}
\end{array}\right),
$$


so that $n$ is the baryon number density in the local rest frame.

With these irreversible currents, of course, the entropy is not conserved. Instead, from Eqs.(8) and (9) with the constraints Eqs. (12) and (13), we have [4]

$$
\partial_{\mu} \sigma^{\mu}=\frac{1}{T}\left(-P^{\mu v} \Pi+\pi^{\mu v}\right) \partial_{\mu} u_{v}-v^{\mu} \partial_{\mu} \alpha
$$

where $\sigma^{\mu}=s u^{\mu}-\alpha v^{\mu}$ is the entropy four-flux, and $\alpha=\mu / T$ and $\mu$ is the chemical potential. The r.h.s. of Eq. (14) is the source term for entropy production.

In non-equilibrium thermodynamics, it is interpreted that entropy production is the sum of the products of thermodynamic forces and irreversible currents. Thus, we can define the scalar, vector and tensor thermodynamic forces,

$$
F=\partial_{\alpha} u^{\alpha}, \quad F_{\mu}=\partial_{\mu} \alpha, \quad F_{\mu v}=\partial_{\mu} u_{v},
$$

respectively. From the second law of thermodynamics, we assume that the entropy production is positive,

$$
\frac{1}{T}\left(-P^{\mu v} \Pi+\pi^{\mu \nu}\right) \partial_{\mu} u_{v}-v^{\mu} \partial_{\mu} \alpha \geq 0
$$

To maintain this algebraic positive definiteness, the most general irreversible currents are given by linear combinations of the thermodynamic forces with the coefficients appropriately chosen. However, if we accept the Curie (symmetry) principle which forbids the mixture of different types of thermodynamic forces [31], the irreversible currents are given by

$$
\begin{aligned}
\Pi & =-\zeta F=-\zeta \partial_{\alpha} u^{\alpha}, \\
\pi_{\mu v} & =\eta P_{\mu v \alpha \beta} \tilde{\pi}^{\alpha \beta}=\eta P_{\mu v \alpha \beta} F^{\alpha \beta}=\eta P_{\mu v \alpha \beta} \partial^{\alpha} u^{\beta}, \\
v_{\mu} & =\kappa P_{\mu v} \tilde{v}^{\mu}=-\kappa P_{\mu \nu} F^{v}=-\kappa P_{\mu v} \partial^{v} \alpha,
\end{aligned}
$$

where $\zeta, \eta$ and $\kappa$ are bulk viscosity, shear viscosity and thermal conductivity coefficients, respectively. Here, $P^{\mu \alpha v \beta}$ is the double symmetric traceless projection,

$$
P^{\mu v \alpha \beta}=\frac{1}{2}\left(P^{\mu \alpha} P^{v \beta}+P^{\mu \beta} P^{v \alpha}\right)-\frac{1}{P_{\lambda}^{\lambda}} P^{\mu v} P^{\alpha \beta},
$$

and we have introduced the quantities $\widetilde{\pi}^{\alpha \beta}$ and $\widetilde{v}^{v}$ which correspond respectively to the shear tensor and irreversible current before the projection.

Eqs.(16) are the prescription of the LL, and it leads to the non-causal propagation of signal. So we should modify these equations to satisfy the relativistic causality principle. The basic point is that the equations of the LL form a parabolic system and we have to convert it to the hyperbolic one. However, at this moment, the generalization of these equation in order to obtain hyperbolic equations is rather self-evident. We introduce the memory function in each irreversible currents, Eq.(16),

$$
\begin{gathered}
\Pi(\tau)=-\int_{-\infty}^{\tau} d \tau^{\prime} G\left(\tau, \tau^{\prime}\right) \zeta \partial_{\alpha} u^{\alpha}\left(\tau^{\prime}\right), \\
\tilde{\pi}^{\mu \nu}(\tau)=\int_{-\infty}^{\tau} d \tau^{\prime} G\left(\tau, \tau^{\prime}\right) \eta \partial^{\mu} u^{\nu}\left(\tau^{\prime}\right), \\
\widetilde{v}^{\mu}(\tau)=-\int_{-\infty}^{\tau} d \tau^{\prime} G\left(\tau, \tau^{\prime}\right) \kappa \partial^{\mu} \alpha\left(\tau^{\prime}\right),
\end{gathered}
$$

where $\tau=\tau(\vec{r}, t)$ is the local proper time. As before, the shear tensor $\pi^{\mu \nu}$ and the irreversible current $\nu^{\mu}$ are then given by the projection of these integrals as

$$
\begin{aligned}
\pi^{\mu \nu} & =P^{\mu v \alpha \beta} \tilde{\pi}_{\alpha \beta}(\tau), \\
v^{\mu} & =P^{\mu v} \widetilde{v}^{\mu}(\tau) .
\end{aligned}
$$

When we start with the finite initial time, say, $\tau_{0}$, the above integrals should read

$$
\begin{gathered}
\Pi(\tau)=-\int_{\tau_{0}}^{\tau} d \tau^{\prime} G\left(\tau, \tau^{\prime}\right) \zeta \partial_{\alpha} u^{\alpha}\left(\tau^{\prime}\right)+e^{-\left(\tau-\tau_{0}\right) / \tau_{R}} \Pi_{0}, \\
\tilde{\pi}^{\mu \nu}(\tau)=\int_{\tau_{0}}^{\tau} d \tau^{\prime} G\left(\tau, \tau^{\prime}\right) \eta \partial^{\mu} u^{v}\left(\tau^{\prime}\right)+e^{-\left(\tau-\tau_{0}\right) / \tau_{R}} \tilde{\pi}_{0}^{\mu \nu}, \\
\widetilde{v}^{\mu}(\tau)=-\int_{\tau_{0}}^{\tau} d \tau^{\prime} G\left(\tau, \tau^{\prime}\right) \kappa \partial^{\mu} \alpha\left(\tau^{\prime}\right)+e^{-\left(\tau-\tau_{0}\right) / \tau_{R} \widetilde{v}_{0}^{\mu}}
\end{gathered}
$$

where $\Pi_{0}, \tilde{\pi}^{\mu v}{ }_{0}$ and $\widetilde{v}^{\mu}{ }_{0}$ are the initial conditions given at $\tau_{0}$.

In Eqs.(18), we have used the same memory function $G$ and consequently a common relaxation time $\tau_{R}$ for the bulk and shear viscosities and heat conduction. We could have used different relaxation times for each irreversible current and this would not alter the basic structure of our theory. However, here we stay with a common relaxation time for all of them for the sake of simplicity. We consider the situation where the time scales of the microscopic degrees of freedom are well separated from those of the macroscopic ones. Then, the effect of the differences of the microscopic relaxation times should not be much relevant in the dynamics described in the macroscopic time scale. Thus we just represent these microscopic time scales in terms of one relaxation time $\tau_{R}$.

The integral expressions (18) are equivalent to the following differential equations,

$$
\begin{aligned}
\Pi & =-\zeta \partial_{\alpha} u^{\alpha}-\tau_{R} \frac{d \Pi}{d \tau}, \\
\tilde{\pi}^{\mu \nu} & =\eta \partial^{\mu} u^{\nu}-\tau_{R} \frac{d \tilde{\pi}^{\mu \nu}}{d \tau}, \\
\widetilde{v}^{\mu} & =-\kappa \partial^{\mu} \alpha-\tau_{R} \frac{d \widetilde{v}^{\mu}}{d \tau},
\end{aligned}
$$

where

$$
\frac{d}{d \tau}=u^{\mu} \partial_{\mu}
$$

is the total derivative with respect to the proper time. The above equations, after the projection (19), can be compared to the corresponding equations in the simplest version of the IS, which is obtained phenomenologically based on extended thermodynamics,

$$
\begin{aligned}
\Pi & =-\frac{1}{3} \zeta_{I S}\left(\partial_{\alpha} u^{\alpha}+\beta_{0} \frac{d \Pi}{d \tau}-\alpha_{0} \partial_{\alpha} v^{\alpha}\right) \\
\pi^{\mu \nu} & =2 \eta_{I S} P^{\mu \alpha v \beta}\left(\partial_{\alpha} u_{\beta}-\beta_{2} \frac{d \pi^{\mu v}}{d \tau}-\alpha_{1} \partial_{\alpha} v_{\beta}\right) \\
v^{\mu} & =-\kappa_{I S} P^{\mu \nu}\left(\frac{n}{\varepsilon+P} \partial_{v} \alpha+\beta_{1} \frac{d v^{v}}{d \tau}+\alpha_{0} \partial_{v} \Pi+\alpha_{1} \partial_{\alpha} \pi_{v}^{\alpha}\right)
\end{aligned}
$$


where $\alpha_{0}, \alpha_{1}, \beta_{0}, \beta_{1}$ and $\beta_{2}$ are constants. Note that the definitions of parameters $\eta, \zeta$ and $\kappa$ are different from that of the IS. Eqs.(23) and (24) have similar aspects. However, our equation is not a special case of the IS. In the IS, the projection operators, which are necessary to satisfy the orthogonality conditions (12) and (13), are included in the differential equations themselves. Thus, it is not possible to derive our equations from the IS. For example, we can write down the differential equation of the heat conduction $v^{\mu}$ by using Eq. (23) as follows,

$$
v^{\mu}=-\kappa P^{\mu v} \partial_{v} \alpha-\tau_{R} \frac{d v^{\mu}}{d \tau}+\frac{d P^{\mu \nu}}{d \tau} \widetilde{v}_{v}
$$

The last two terms of the above equation do not appear in the IS. In addition, there appear the extra coupling terms among dissipative terms (those with coefficients $\alpha_{0}$ and $\alpha_{1}$ ) which, in our approach, are not included based on the Curie principle.

In spite of these differences, our equations are found to be still hyperbolic in the linearized form. When we consider the propagation of small perturbations on the homogeneous and static background, the projection operator turns out to be a constant matrix. Therefore we can easily see that our linearized equation of motion has the same structure as the IS with $\alpha_{0}=\alpha_{1}=0$. Thus the speed of pulse propagation is finite as discussed by Hiscock-Lindblom [32-34].

Till now, we have considered that the relaxation time $\tau_{R}$ is constant. However in practical problems, it is a function of thermodynamical variables. Then the memory function should be generalized as

$$
G\left(\tau, \tau^{\prime}\right) \rightarrow \frac{1}{\tau_{R}\left(\tau^{\prime}\right)} e^{-\int_{\tau^{\prime}}^{\tau} \frac{1}{\tau_{R}\left(\tau^{\prime \prime}\right)} d \tau^{\prime \prime}}
$$

\section{ENTROPY PRODUCTION}

It should be emphasized that our theory is not a simplified version of the IS but there exists an essential difference for the treatment of the entropy production term. The IS requires the general algebraic form of the non-negative definite expression for entropy production following non-equilibrium thermodynamics. In our approach, we have relaxed this condition, that is, the expression Eq.(15) for the entropy production

$$
\frac{1}{T}\left(-P^{\mu v} \Pi+\pi^{\mu v}\right) \partial_{\mu} u_{v}-v^{\mu} \partial_{\mu} \alpha \geq 0
$$

does not guarantee algebraically owing to the non-locality in time contained in $\Pi, \pi^{\mu \nu}$ and $v^{\mu}$ through Eq.(18). This might seem to be dangerous. However, strictly speaking, the increase of entropy is essentially a concept in equilibrium thermodynamics and the requirement of positiveness should apply only to thermal equilibrium states. As a matter of fact, it was recently shown that the entropy absorption process can occur in the non-equilibrium processes of mesoscopic systems [35]. In our approach, we are dealing with a fluid element which is out of equilibrium, interacting with the neighboring elements. Therefore, within the relaxation time, its entropy content may increase or decrease depending on the dynamics and its time scales. Thus, the requirement of the algebraic positive definiteness irrespective of any field configuration seems to be too restrictive. The requirement of non-negative entropy production may be relaxed for far-from-equilibrium states. In our case, apart from the projection operators, the expression for entropy production has the form

$$
Q(\tau)=f(\tau) \frac{1}{\tau_{R}} \int^{\tau} d \tau^{\prime} e^{-\left(\tau-\tau^{\prime}\right) / \tau_{R}} f\left(\tau^{\prime}\right),
$$

where $f$ is one of $\partial_{\mu} u_{v}$ or $\partial_{\mu} \alpha$. For small $\tau_{R}$, we may expand $f\left(\tau^{\prime}\right)$ near $\tau$,

$$
f\left(\tau^{\prime}\right)=f(\tau)-\left(\tau-\tau^{\prime}\right) \frac{d f(\tau)}{d \tau}+\cdots,
$$

and we have

$$
Q(\tau)=f(\tau)\left[f(\tau)-\tau_{R} \frac{d f(\tau)}{d \tau}+O\left(\tau_{R}^{2}\right)\right] .
$$

Thus, as far as

$$
\left|\tau_{R} \frac{d f(\tau)}{d \tau}\right|<|f(\tau)|,
$$

the positiveness of the entropy is ensured. The 1.h.s. of the above equation is the amount of variation of $f(\tau)$ within a small time interval $\tau_{R}$. Thus, the above condition shows that if the time evolution of the system is not too violent (the change of field values within the relaxation time is less than its value), then the local entropy production is not negative. For the example discussed below, we can show explicitly the positive definiteness of entropy production on our formulation.

In the above, we considered the relaxation time as constant just for illustration. The similar conclusion can be derived when the variation of the relaxation time is not so violent.

\section{PROPAGATION SPEED}

We discuss the propagation speed of the simple $1+1$ dimensional system. For simplicity, we set $n=0$. We consider the small perturbation of the velocity filed $\delta u^{\mu}$,

$$
u^{\mu}=u_{0}^{\mu}+\delta u^{\mu},
$$

where

$$
u_{0}^{\mu}=(1,0) .
$$

Then, the shear viscosity disappears. The equation of the energy density is given by the following coupled equation,

$$
\left(\begin{array}{ccc}
i \omega & -i k(\varepsilon+p) & 0 \\
\alpha(-i k) & i \omega(\varepsilon+p) & -i k \\
0 & -i k \zeta & 1+\tau_{R} \gamma i \omega
\end{array}\right)\left(\begin{array}{c}
\delta \varepsilon \\
\delta U^{1} \\
\delta \Pi
\end{array}\right)=0
$$

where we assumed $\delta p=\alpha \delta \varepsilon$.

To have non-trivial solutions, $\omega$ should satisfy the following dispersion relation,

$$
\omega=x+\frac{\bar{p}}{3} \frac{1}{x}+\frac{i}{3 \tau_{R}},
$$


where

$$
\begin{aligned}
& x=(-i)^{1 / 3} \sqrt{\frac{q}{2}+\sqrt{\left(\frac{q}{2}\right)^{2}+\left(\frac{\bar{p}}{3}\right)^{3}}}, \\
& \bar{p}=\left(\frac{\zeta}{\tau_{R}} \frac{1}{\varepsilon+p}+\alpha\right) k^{2}-\frac{1}{3 \tau_{R}^{2}}, \\
& q=\frac{1}{3 \tau_{R}}\left(2 \alpha-\frac{\zeta}{\tau_{R}} \frac{1}{\varepsilon+p}\right) k^{2}+\frac{2}{\left(3 \tau_{R}\right)^{3}} .
\end{aligned}
$$

The asymptotic forms of the solution of $x$ for large $k$ are given by

$$
x \rightarrow O(1), \sqrt{\frac{\zeta}{\tau_{R}} \frac{1}{\varepsilon+p}+\alpha k},-\sqrt{\frac{\zeta}{\tau_{R}} \frac{1}{\varepsilon+p}+\alpha k} .
$$

Thus, the asymptotic form of the dispersion relation is

$$
\omega= \pm \sqrt{\frac{\zeta}{\tau_{R}} \frac{1}{\varepsilon+p}+\alpha k}-i \frac{1}{3 \tau_{R}} .
$$

When we ignore the imaginary part as is done in Ref. [26, 36], the phase velocity is given by

$$
v_{p h}=\sqrt{\frac{\zeta}{\tau_{R}} \frac{1}{\varepsilon+p}+\alpha}
$$

It is clear that the phase velocity becomes infinite at the limit of $\tau_{R} \rightarrow 0$. If we can identify the phase velocity with the speed of propagation, we can conclude that our theory can satisfy causality by choosing the parameters.

\section{ONE-DIMENSIONAL SCALING SOLUTION}

To see how the above scheme works, let us apply it to the one dimensional scaling solution of the Bjorken model. This has been studied already in the framework of the IS [19-23]. The components of the irreversible currents is written down explicitly as

$$
\begin{aligned}
& \Pi(\tau)=-\int_{\tau_{0}}^{\tau} d \tau^{\prime} G\left(\tau, \tau^{\prime}\right) \frac{\zeta}{\tau^{\prime}}+\tau_{R}\left(\tau_{0}\right) G\left(\tau, \tau_{0}\right) \Pi\left(\tau_{0}\right) \\
& \Omega(\tau)=-\int_{\tau_{0}}^{\tau} d \tau^{\prime} G\left(\tau, \tau^{\prime}\right) \frac{\eta}{\tau^{\prime}}+\tau_{R}\left(\tau_{0}\right) G\left(\tau, \tau_{0}\right) \Omega\left(\tau_{0}\right) \\
& \Phi(\tau)=-\int_{\tau_{0}}^{\tau} d \tau^{\prime} G\left(\tau, \tau^{\prime}\right) \kappa \frac{d \alpha}{d \tau^{\prime}}+\tau_{R}\left(\tau_{0}\right) G\left(\tau, \tau_{0}\right) \Phi\left(\tau_{0}\right) \\
& \left(\tilde{\pi}_{\mu v}\right)=-\left(\begin{array}{cc}
-\sinh ^{2} y & \sinh y \cosh y \\
\sinh y \cosh y & -\cosh ^{2} y
\end{array}\right) \Omega(\tau) \\
& \left(\tilde{\mathrm{v}}_{\mu}\right)=\left(\begin{array}{c}
\cosh y \\
-\sinh y
\end{array}\right) \Phi(\tau)
\end{aligned}
$$

where we have used the hyperbolic variables,

$$
t=\tau \cosh y, x=\tau \sinh y,
$$

and used the scaling ansatz in $y$ (that is, there is no $y$ dependence in thermodynamic variables). $\Pi\left(\tau_{0}\right), \Omega\left(\tau_{0}\right)$ and $\Phi\left(\tau_{0}\right)$ are initial values for $\Pi(\tau), \Omega(\tau)$ and $\Phi(\tau)$. We obtain

$$
\begin{aligned}
\pi^{\mu \nu} & =P^{\mu \alpha v \beta} \tilde{\pi}_{\alpha \beta}=-\frac{2 \Omega}{3} P^{\mu v}, \\
v^{\mu} & =P^{\mu v} \tilde{v}_{\mu}=0 .
\end{aligned}
$$

As we see, in the one-dimensional case, if $\zeta$ and $\eta$ are proportional as functions of thermodynamic quantities such as $T$ and $\mu$, then the bulk and shear viscosity terms are not independent, and

$$
\Pi \propto \Omega .
$$

However, when $\zeta$ and $\eta$ have, in general, different dependence on the thermodynamic quantities, the two viscosities act differently.

The time component of the divergence of $T^{\mu \nu}$ gives

$$
\frac{d}{d \tau} \varepsilon(\tau)+\frac{\varepsilon+P+\Pi}{\tau}+\frac{2}{3} \frac{\Omega}{\tau}=0
$$

The equation for the space component is automatically satisfied by the scaling ansatz showing its consistency. The entropy production rate is calculated to be

$$
\partial_{\mu}\left(s u^{\mu}-\alpha v^{\mu}\right)=-\frac{1}{T} \frac{1}{\tau}\left(\Pi+\frac{2}{3} \Omega\right)
$$

Since $\Pi$ and $\Omega$ are negative definite, the entropy production is positive definite.

\section{A. Solutions}

When $\zeta=\zeta_{0}, \eta=\eta_{0}$ and $\tau_{R}$ are constant, then we can obtain analytic expression for the proper energy density. We obtain

$$
\Omega=\frac{\eta_{0}}{\zeta_{0}} \Pi=-\frac{\eta_{0}}{\tau_{R}} e^{-\frac{\tau}{\tau_{R}}}\left[\operatorname{Ei}\left(-\frac{\tau}{\tau_{R}}\right)-\operatorname{Ei}\left(-\frac{\tau_{0}}{\tau_{R}}\right)+E_{0}\right],
$$

where

$$
\operatorname{Ei}(-x)=\int_{x}^{\infty} \frac{e^{-t}}{t} d t
$$

is the exponential integral, and $E_{0}$ is a constant which should be determined from the initial condition for $\Pi$ (or $\Omega$ ). For a relativistic ideal gas,

$$
P=\frac{\varepsilon}{3}
$$

we get

$$
\frac{d \varepsilon}{d \tau}+\frac{4}{3} \frac{\varepsilon}{\tau}+\left(\frac{2 \eta_{0}}{3 \zeta_{0}}+1\right) \frac{\Pi(\tau)}{\tau}=0,
$$

so that for $E_{0}=0$,

$$
\varepsilon(\tau)=\varepsilon_{0}\left(\frac{\tau_{0}}{\tau}\right)^{4 / 3}\left[1-\frac{1+2 \eta_{0} / 3 \zeta_{0}}{\varepsilon_{0} \tau_{0}^{4 / 3}} \int_{\tau_{0}}^{\tau} d t t^{1 / 3} \Pi(t)\right],
$$


where the integral containing the exponential function can still be evaluated analytically. The temperature is determined from the energy density as

$$
\varepsilon=\sigma_{S B} T^{4}
$$

where $\sigma_{S B}$ is the Stephan-Boltzmann constant.

On the other hand, a typical estimate from the kinetic theory shows that the shear viscosity $\eta$ is proportional to the entropy density $s, \eta=b s$, where $b$ is a constant [20, 22]. Following Ref. [22], we choose $b=1.1$. Furthermore, we use the relaxation time $[20,22]$

$$
\tau_{R}=\frac{3 \eta_{I S}}{4 p}=\frac{3 \eta}{8 p}
$$

Here, it should be noted that our definition of $\eta$ is twice of other papers [19-23]. The effect of the bulk viscosity has not been discussed in previous papers. We analogously assume that the bulk viscosity has the similar $s$ dependence, $\zeta=a s$. For a baryon free relativistic gas, $s$ is related as the energy density as

$$
s=C \varepsilon^{3 / 4}
$$

so that the equation for the energy density becomes

$$
\tau \frac{d^{2} \varepsilon}{d \tau^{2}}+\left(\frac{7}{3}+\frac{\tau}{\tau_{R}}\right) \frac{d \varepsilon}{d \tau}+\frac{1}{\tau_{R}}\left(\frac{4}{3} \varepsilon-C^{\prime} \frac{\varepsilon^{3 / 4}}{\tau}\right)=0
$$

were

$$
C^{\prime}=\left(a+\frac{2}{3} b\right) C
$$

The above equation is the same as the equation derived in Ref. [22]. It should be noted that this coincidence is due to the specific property of this particular model. In Ref. [22], the above equation is obtained under the assumption of no acceleration,

$$
\frac{d}{d \tau} u^{\mu}=0
$$

which is automatically satisfied in the scaling solution. For general cases, our theory does not require such a condition at all to be applied. Eq.(53) can be solved for the initial condition,

$$
\varepsilon=\varepsilon\left(\tau_{0}\right)
$$

and

$$
\left.\frac{d \varepsilon}{d \tau}\right|_{\tau=\tau_{0}}=-\frac{1}{\tau_{0}}\left[\frac{4}{3} \varepsilon_{0}+\Pi\left(\tau_{0}\right)+\frac{2}{3} \Omega\left(\tau_{0}\right)\right] .
$$

Now, we show our numerical results. To compare to previous works, we ignore the bulk viscosity. In Fig. 1, we show the energy density $\varepsilon$ obtained by solving Eq.(53) as function of proper time $\tau$. As the initial condition, we set $\varepsilon\left(\tau_{0}\right)=1 \mathrm{GeV} / \mathrm{fm}^{3}, \Pi\left(\tau_{0}\right)=\Omega\left(\tau_{0}\right)=0$ at the initial proper time $\tau_{0}=0.1 \mathrm{fm} / \mathrm{c}$. The first two lines from the top represents

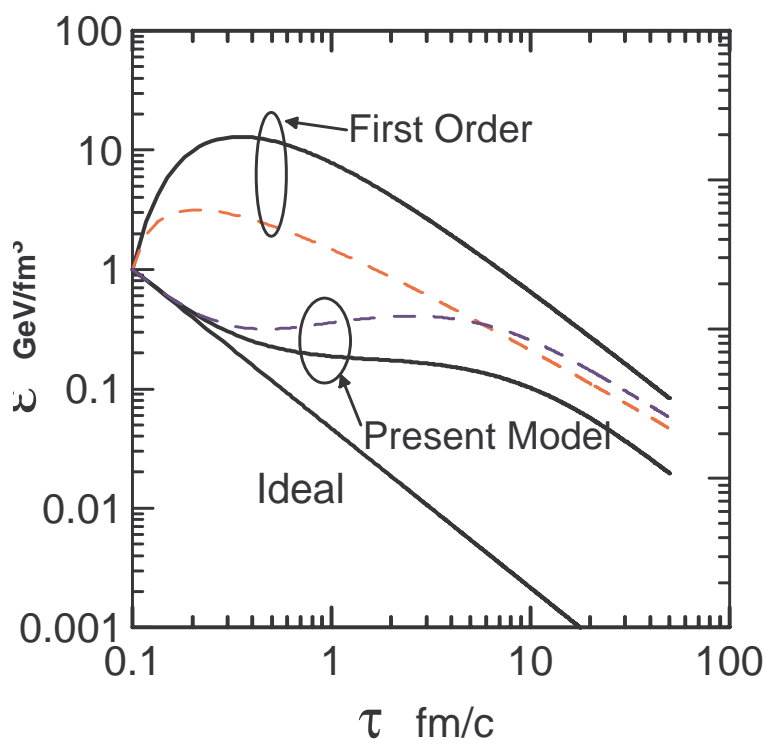

FIG. 1: (Color online) The time evolution of the energy density. The dashed curves correspond to the calculations with the constant viscosity and relaxation time. The first two lines from the top represents the results of the LL. Next two lines shows the results of our theory. The last line is the result of ideal hydrodynamics.

the results of the LL. The next two lines show the results of our theory. The last line is the result of ideal hydrodynamics. For the solid lines, we calculated with the viscosity and relaxation time which depend on temperature. Initially, the effect of viscosity is small because of the memory effect, the behavior of our theory is similar to that of ideal hydrodynamics. After the time becomes larger than the relaxation time, the memory effect is not effective anymore and the behavior is similar to the result of the LL. As we have mentioned, the behavior of our theory is the same as the result obtained in Ref. [22] in this case. For the dashed lines, we calculated with the constant viscosity and relaxation time, $\eta=\eta\left(\varepsilon_{0}\right)$ and $\tau_{R}=\tau_{R}\left(\varepsilon_{0}\right)$. In this case, the viscosity is constant so that the heat production stays longer and has a smaller slope as a function of time asymptotically.

Sometimes the emergence of the initial heat-up in the LL (the dashed curve in Fig.1) is interpreted as an intrinsic problem of the first order theory. However, such behavior can also appear even in the second order theory. In Fig. 2, we set $\Pi\left(\tau_{0}\right)=\zeta\left(\tau_{0}\right) / \tau_{0}$ and $\Omega\left(\tau_{0}\right)=\eta\left(\tau_{0}\right) / \tau_{0}$ as the initial conditions. In particular, the initial heat-up also appears in the second order theory depending on the initial condition for the irreversible currents (see Fig. 2). Therefore, this heat-up is not the problem of the first order theory but rather the specific property of the scaling ansatz. This was already pointed out by Muronga [20]. The physical reason for this heat-up is due to the use of the Bjorken solution for the velocity field. In this case, the system acts as if an external force is applied to keep the velocity field as a given function of $\tau$. Thus, depending on the relative intensity of the viscous terms compared to the pressure, the external work converted to the local heat 


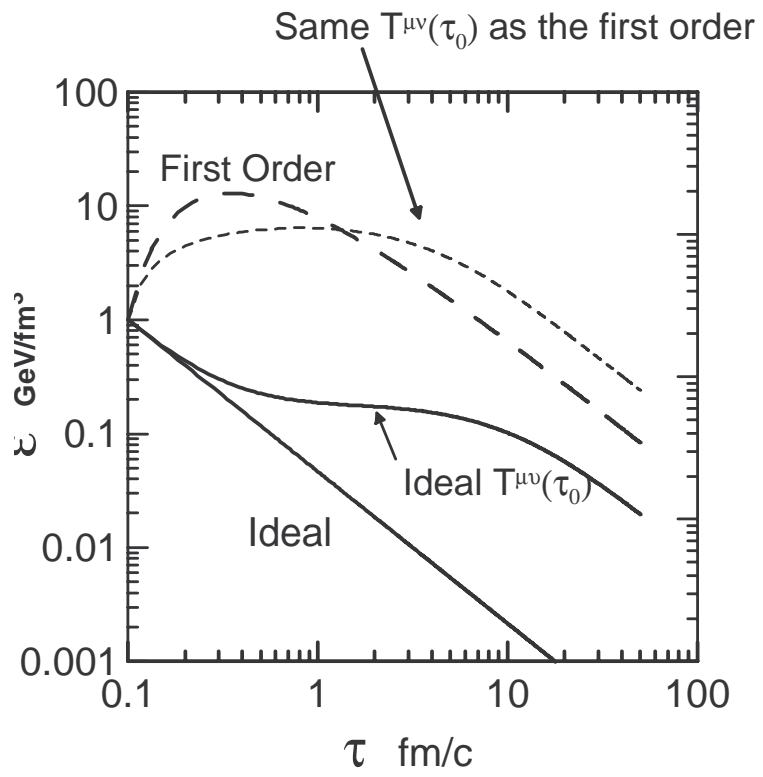

FIG. 2: The time evolution of energy density with the different initial conditions from Fig. 1. The dashed and short dashed lines represent the result of the LL and our theory, respectively . For comparison, our result of Fig. 1 is shown, again (ideal $T^{\mu v}\left(\tau_{0}\right)$ ). The last line from the top is the result of ideal hydrodynamics. In this case, the energy heat-up is observed even in our theory.

production can overcome the temperature decrease due to the expansion.

\section{SUMMARY AND CONCLUDING REMARKS}

In this paper, we proposed an alternative approach to this question, different from the IS. We start from the physical analysis of the irreversible currents according to the LandauLifshitz theory. Then, the irreversible currents are given by integral expressions which take into account the relaxation time. In this way, causality is recovered and at the same time a simple physical structure of the LL is preserved. In our approach, only one additional parameter was introduced, the relaxation time, $\tau_{R}$. The resulting equation of motion then becomes hyperbolic and causality can be restored [5]. Naturally, causality depends on the choice of the values of the parameters including the relaxation time within the framework of this approach.
More specifically, we verified that the linearized equation of motion for small perturbations in the homogeneous, static background coincides with Hiscock-Lindblom [32-34] except for the coupling among the different irreversible currents. These couplings are not included in our theory considering the Curie principle. Of course the Curie principle is believed to be valid in the regime of the first order theory and in the second order regime these couplings might be present. However, the existence of the Curie principle may imply that these couplings are small compared with the direct terms.

The essential difference of our formalism from the IS is the expression for entropy production. In the IS, entropy production is required to be positive definite algebraically. Thus, the integral form like our formulation is not possible even neglecting some coupling terms. We relaxed this condition, that is, the positiveness of entropy production is required only for hydrodynamical motion with time scales longer than the relaxation time. For extremely violent change of variables, the instantaneous entropy change for a hydrodynamic cell would not necessarily be positive definite.

We have applied our theory to the case of the onedimensional scaling solution of Bjorken and obtained the analogous behavior of previous analysis. In this case we can prove explicitly the positiveness of entropy production. We showed the time evolution of the temperature. As expected, our theory gives the same result of Ref. [22], because the no-acceleration condition used in Ref. [22] is automatically satisfied in this model. Note that our theory is applicable to more general case where the acceleration is important.

The transport coefficients contained in relativistic dissipative hydrodynamics should in principle be calculated from QCD. In the first order theory, it is known that the transports coefficients can be calculated by the Kubo formula. However, this formula does not gives the transport coefficients in the second order theory, as is shown in Ref. [27] explicitly and the corresponding corrections should be evaluated.

Our theory is particularly adequate to be applied to the hydro-code such as SPheRIO which is based on the Lagrangian coordinate system $[17,18]$. Implementation of the present theory to the full three-dimensional hydrodynamics is now in progress.

Authors are grateful for fruitful discussion with C. E. Aguiar, E.S. Fraga, T. Hirano and T. Hatsuda. T. Koide would like to thank David Jou for useful comments. This work was partially supported by FAPERJ, FAPESP, CNPq and CAPES.
[1] T. Kodama, T. Koide, G. S. Denicol, and Ph. Mota, hep$\mathrm{ph} / 0606161$.

[2] D. Teaney, Phys. Rev. C 68, 034913 (2003).

[3] C. Eckart, Phys. Rev. 58, 919 (1940).

[4] L. D. Landau and E. M. Lifshitz, Fluid Mechanics (Pergamon, New York, 1959).

[5] I. Müller, Living Rev. Rel. 2, 1 (1999).

[6] D. Jou, J. Casas-Vázquez, and G. Lebon, Rep. Prog. Phys. 51, 1105 (1988); Rep. Prog. Phys. 62, 1035 (1999),
[7] I. Müller, Z. Phys. 198, 329 (1967).

[8] W. Israel, Ann. Phys. (N.Y.) 100, 310 (1976).

[9] J. M. Stewart, Proc. Roy. Soc. A 357, 59 (1977).

[10] W. Israel and J. M. Stewart, Ann. Phys. (N.Y.) 118, 341 (1979).

[11] H. Grad, Commun. Pure Appl. Math. 2, 331 (1949).

[12] I. Liu, I. Müller, and T. R, Ann. Phys. (N.Y.) 169, 191 (1986).

[13] R. Geroch and L. L. Phys. Rev. D 41, 1855(1990).

[14] R. Geroch and L. Lindblom, Phys. Rev. D 41, 1855 (1990).

[15] G. Nagy and O. Reula, J. Phys. A 30, 1695 (1997). 
[16] E. Calzetta, Class. Quant. Grav. 15, 653 (1998).

[17] C. E. Aguiar, T. Kodama, T. Osada, and Y. Hama, J. Phys. G 27, 75 (2001).

[18] Y. Hama, T. Kodama, and O. Socolowski, Braz. J. Phys. 35, 24 (2005).

[19] A. Muronga, Phys. Rev. Lett. 88, 062302 (2002); ibid 89, 159901(E) (2002).

[20] A. Muronga, Phys. Rev. C 69, 034903 (2004).

[21] A. Muronga and D. H. Rischke, nucl-th/0407114.

[22] R. Baier, P. Romatschke, and U. A. Wiedemann, Phys. Rev. C 73, 064903 (2006).

[23] R. Baier, P. Romatschke, and U. A. Wiedemann, nuclth/0604006.

[24] C. Cattaneo, Atti del Semin. Mat. e Fis. Univ. Modena 3, 3 (1948).

[25] P. M. Morse and H. Feshbach, Methods of Theoretical Physics, p. 865 (McGraw Hill, New York, 1953).

[26] T. Koide, G. Krein, and R. O. Ramos, Phys. Lett. B 636, 96 (2006).
[27] T. Koide, Phys. Rev. E 72, 026135 (2005)

[28] M. A. Aziz and S. Gavin, Phys. Rev. C 70, 034905 (2004).

[29] Such a memory function was also used in the hydrodynamic equations to take into account the time delay necessary to thermalize the micro turbulences in a large scale system such as supernova explosion. See T.Kodama, R. Donangelo and M. Guidry, Int. J. Mod. Phys. C9, 745 (1998).

[30] T. Koide, G. S. Denicol, Ph. Mota, and T. Kodama, hep$\mathrm{ph} / 0909117$.

[31] See, for example, T. Koide, J. Phys. G 31, 1055 (2005).

[32] W. A. Hiscock and L. Lindblom, Ann. Phys. (N.Y.) 151, 466 (1983).

[33] W. A. Hiscock and L. Lindblom, Phys. Rev. D 31, 725 (1985).

[34] W. A. Hiscock and L. Lindblom, Phys. Rev. D 35, 3723 (1987).

[35] U. Seifert, Phys. Rev. Lett. 95, 040602 (2005).

[36] Strictly speaking, the phase velocity does not necessarily give the propagation speed. See [25]. 